\title{
La région métropolitaine européenne Zurich-Bâle : recompositions des fonctions stratégiques dans le cadre de la mondialisation
}

The European metropolitan region Zurich-Basle : rethinking urban systems as a result of globalization

Europäische Metropolregion Zürich-Basel : Umwertungen im Städtesystem als Folge der Globalisierung

\section{Heiko Behrendt et Christian Kruse}

\section{OpenEdition}

Journals

Édition électronique

URL : http://journals.openedition.org/rge/2817

DOI : $10.4000 /$ rge.2817

ISSN : 2108-6478

Éditeur

Association des géographes de l'Est

Édition imprimée

Date de publication : 1 janvier 2002

ISSN : 0035-3213

\section{Référence électronique}

Heiko Behrendt et Christian Kruse, « La région métropolitaine européenne Zurich-Bâle

recompositions des fonctions stratégiques dans le cadre de la mondialisation », Revue Géographique de l'Est [En ligne], vol. 42 / 1-2 | 2002, mis en ligne le 05 janvier 2011, consulté le 08 septembre 2020. URL : http://journals.openedition.org/rge/2817 ; DOI : https://doi.org/10.4000/rge.2817

Ce document a été généré automatiquement le 8 septembre 2020

Tous droits réservés 


\section{La région métropolitaine européenne Zurich-Bâle : recompositions des fonctions stratégiques dans le cadre de la mondialisation}

The European metropolitan region Zurich-Basle : rethinking urban systems as a result of globalization

Europäische Metropolregion Zürich-Basel : Umwertungen im Städtesystem als Folge der Globalisierung

Heiko Behrendt et Christian Kruse

\section{Introduction : un noyau métropolitain et un centre dans une constellation polynucléaire?}

1 L'affirmation «Bâle est à la fois le cœur de la région transfrontalière du Rhin supérieur méridional et partie intégrante de la région métropolitaine européenne de Zurich » est osée dans la mesure où elle est difficile à justifier empiriquement (voire aussi autrement); seules des évaluations scientifiques et des développements politiques seraient à même de faire progresser un tel discours. Elle requiert aussi la supposition selon laquelle le nom ou plutôt le «label Zurich » est lié à un lieu international plus important que celui de Bâle, chacun étant doté de métafonctions relevant habituellement des métropoles européennes (Zürcher Nationalbank, 2000 ; RECLUS, 1989). On n'a cependant clarifié ni les limites du développement spatial, ni la différenciation fonctionnelle de Zurich et de Bâle. Aussi les propos des auteurs ne portent-ils pas, à vrai dire, sur l'appartenance de Bâle et de son agglomération à la région métropolitaine de Zurich - réelle ou non -; ils prennent en compte un 
cadre préalablement défini et s'interrogent sur les recompositions des fonctions entre les centres.

2 La notion de région métropolitaine est en effet un nouveau concept issu de la réflexion sur de nouvelles perspectives de l'aménagement du territoire en Allemagne et dans l'Union européenne. En Allemagne, le concept d'aménagement retenu depuis 1995 et établi officiellement par le ministère fédéral de l'aménagement du territoire prend en compte le rayonnement des pôles au-delà de leurs limites régionales administratives, tant à l'échelle nationale qu'internationale. Ce concept, repris par l'Union européenne, doit inciter les chercheurs et aménageurs à se préoccuper des phénomènes relatifs aux répercussions de la mise en réseau d'espaces fonctionnels. Il importe de définir, à la lumière d'enquêtes appropriées, la nature et le degré de l'évolution de ces régions métropolitaines, leurs aires de chalandise autant que leurs articulations pendulaires, avant d'être à même de définir les limites territoriales précises du mouvement en cours.

En Suisse, cette notion n'est pas reconnue institutionnellement comme région d'aménagement. Le gouvernement confédéral a lancé par contre depuis les années 1990 le concept de métropole suisse, qui serait en fait une région urbaine polycentrique composée de centres urbains spécialisés fonctionnant en réseau. Dans ce contexte, toutes les villes suisses doivent fonctionner comme un espace à mobilité élevée dans lequel la cadence et la durée des liaisons sont à optimiser ; le concept « Train 2000 » est orienté dans ce sens. Mettant à profit ces approches nationales, on tente ici d'appliquer à Zurich le concept de région métropolitaine tel qu'il est évoqué ci-dessus. Les auteurs ont relevé les critères qu'il est possible de retenir actuellement pour la définition de la région métropolitaine de Zurich en gestation. Leur effort ne pouvant porter sur une délimitation exacte de celle-ci, ils en dégagent les tendances (fig. 1), focalisant principalement leur attention sur le rôle de Bâle dans cette construction territoriale. Dans ce contexte, il est entendu que la notion «Zurich, downtown Swizzerland » (voir sous 1) n'est pas un concept d'aménagement du territoire, mais une notion fondée sur la préoccupation de rapprocher l'ensemble des villes du pays dans le cadre d'un concept mettant à profit les nouvelles potentialités offertes par une mobilité généralisée. 


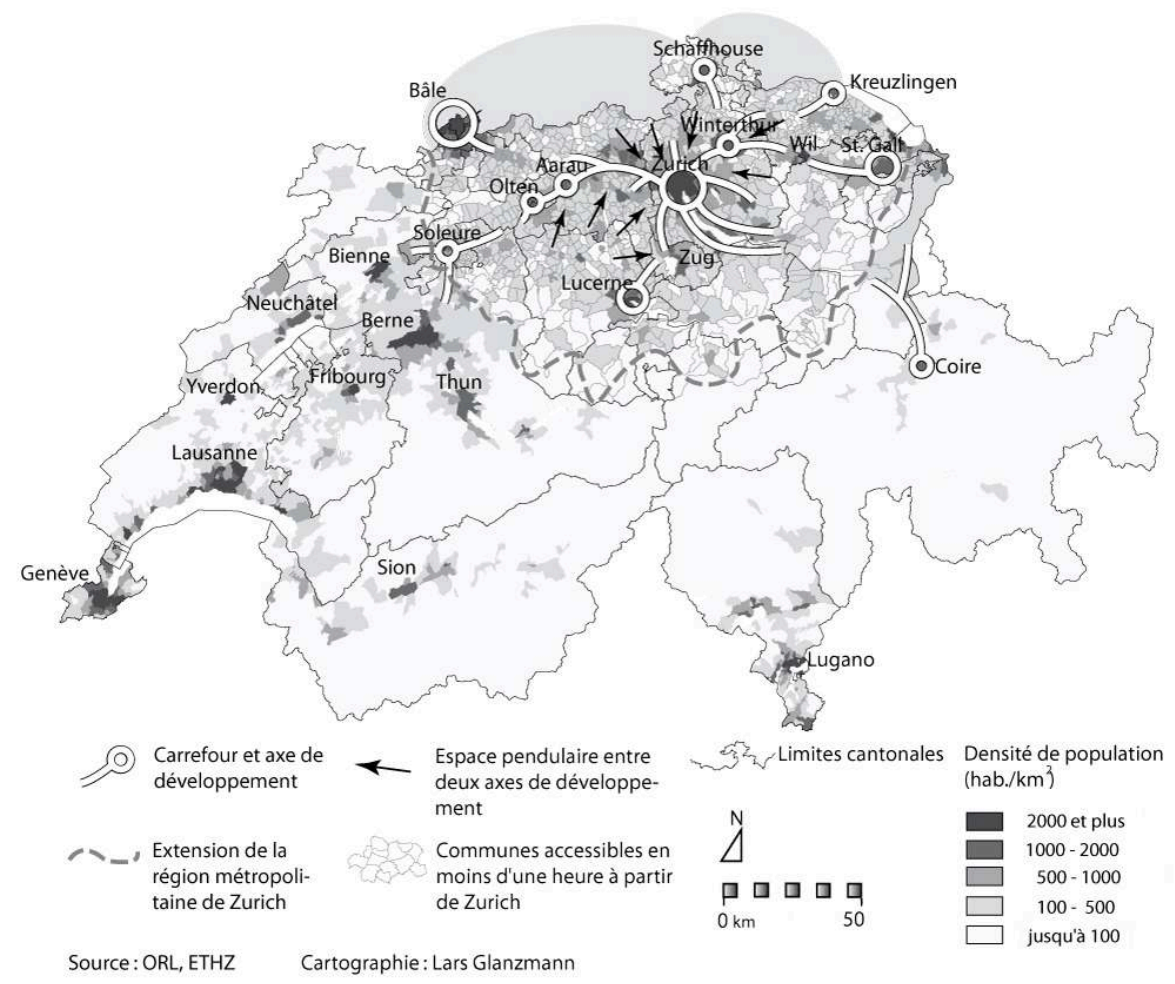

\section{Des discours incertains}

4 L'examen du discours tel qu'il est produit dans les médias et par les élus fait apparaître très vite que celui-ci est superficiel. Il ressemble à tous ceux menés dans de nombreux débats politiques, qui visent à éviter toute justification et toute concrétisation. Il suffit d'observer les tentatives de la ville de Zurich pour se hisser au niveau métropolitain dans le cadre de la compétition internationale, pour prendre conscience des difficultés qui sont inhérentes à pareille entreprise. Etudes et analyses (Zürcher Nationalbank, 2000) révèlent le problème posé, à savoir d'une part qu'une base statistique suffisante dépassant le canton de Zurich fait défaut, et d'autre part, qu'il est difficile de définir une métropole qui dépasse largement le canton en tant qu'espace économique et qui englobe également Bâle entre autres villes.

5 Ces réflexions, appuyées sur l'image "Zurich, downtown Switzerland », comptent aussi plus ou moins pour l'organisme de marketing «Greater Zurich Area - The Zurich Network (GZA) ", créé en 1999, selon le modèle d'autres régions métropolitaines en Europe, à des fins de représentation des intérêts en vue du développement économique de la grande région de Zurich. Celle-ci est l'expression d'une coopération de la ville de Zurich, des cantons environnants - surtout ceux jouxtant la frontière germano-suisse et ceux localisés entre Zurich et Bâle (fig. 1) -, ainsi que des représentants de la multitude de firmes ayant comme support des réseaux de petites et moyennes entreprises. Le GZA définit son rayon d'action en vue d'implanter des firmes étrangères intéressées à l'intérieur d'un cercle où la distance automobile par rapport à l'aéroport de Zurich-Kloten est de 60 à 90 minutes. Indépendamment du fait que certains des cantons situés à l'intérieur de ce périmètre ont déjà signalé clairement leur refus de 
participer au GZA, les arguments clairs susceptibles de témoigner en faveur d'une unité fonctionnelle de la Grande Région de Zurich et de l'appartenance de Bâle à celle-ci font également défaut.

6 Les incertitudes résident dans l'absence d'études menées sur les fonctions urbaines avec comme idée que Bâle appartient à la région métropolitaine de Zurich, ce qui rend aléatoire toute approche sérieuse. Comme le concept de Bâle demeure globalement vague, on ne sait pas de quel «Bâle» il est question. C'est ce qui explique que dans le discours quotidien, les uns ont recours à l'expression aventureuse «Bâle appartient à Zurich », les autres sont conduits à nier carrément toute possibilité d'appartenance.

\section{Les indispensables différenciations}

$7 \quad \mathrm{Au}$ point de vue scientifique, il est important d'analyser ces structures régionales, dans la mesure où le thème soulevé pose de multiples questions d'ordre socio-économique et politique (OCDE, 2000). Répondre à ces questions devient urgent, dans la mesure où le développement d'initiatives régionales se renforce, dans le cadre de l'idée de gouvernance, sous la forme de groupements qui prennent en charge des problèmes habituellement dévolus aux milieux politiques sans en avoir la charge officielle. Avant d'aborder de façon générale la problématique relative au présent article, clarifions quelques préalables indispensables à la compréhension de notre démarche.

Dans le contexte des régions de Bâle et de Zurich (fig. 1), il s'agit, en premier lieu, de deux systèmes régionaux différents qui révèlent de nouvelles formes et fonctions de la division spatiale du travail. Il convient de préciser nettement qu'il importe d'éviter de porter un jugement de valeur sur les différences qualitatives de ces systèmes régionaux. Il ne s'agit pas d'un classement de ces deux régions. Il est par contre important de penser de manière systémique et de tenir compte du fait que des systèmes internationaux, nationaux et régionaux accomplissent des fonctions différentes.

9 En second lieu, il faut prendre en considération la dimension temporelle sous la forme d'une revalorisation fonctionnelle à l'intérieur du système urbain suisse, mais aussi international. Dans la perception politique, l'intérêt de ce processus est indiscutable. Celui-ci est prévu par le texte officiel relatif aux «fondements de l'aménagement du territoire de la Suisse ", adopté par le parlement fédéral en 1996 (Bundesamt für Raumplanung, 1996). Jusqu'à présent, rien n'a toutefois été entrepris pour le faire progresser en vue d'en connaître les répercussions politiques. Cette situation est pourtant courante dans de nombreux Etats.

10 En troisième lieu, cette nouvelle démarche contribue à modifier des comportements politiques trop habitués à un système spatial simple aux territoires nettement délimités. Dans cette optique, il faudrait aussi réformer des procédés administratifs dépassés, ainsi que des habitudes trop traditionnelles prises en matière d'aménagement du territoire à l'échelle régionale.

11 En quatrième lieu, il convient de préciser que c'est justement cette situation régionale qui ne correspond à aucun phénomène national. Les problèmes posés depuis l'échelle locale jusqu'à l'échelle nationale, et qui constituent des caractéristiques fondamentales des systèmes fédéraux, subissent en effet des modifications par suite de multiples influences internationales. Cette influence exogène renforcée reflète ainsi le niveau 
européen du nouveau paysage politique, dont les ressorts centraux s'expriment à travers le système des métropoles régionales européennes (Dielemann et Faludi, 1998). Il n'est pas nécessaire d'être membre de l'Union européenne pour participer à ce mouvement. Des développements économiques mondiaux ont devancé depuis longtemps les systèmes politiques en place pour contribuer à susciter de façon marquante des structures fonctionnelles régionales dans un cadre polycentrique.

12 A la lumière de ces préalables il apparaît très vite de façon claire que la structure systémique des régions bâloise et zurichoise ne peut pas être représentée par un modèle simpliste selon lequel il est possible de définir le degré d' "appartenance » de l'une à l'autre. C'est pourquoi la présente contribution met en relief une possible appartenance de Bâle à une structure systémique de la région métropolitaine européenne de Zurich ; dans cette démarche l'« autonomie » d'une région bâloise sur le Rhin supérieur est parfaitement acceptée, dans la mesure où celle-ci accomplit encore d'autres fonctions.

\section{Le concept de région métropolitaine européenne}

L'évolution politique et spatiale de l'Europe s'effectue de plus en plus sur la base d'une mise en réseaux de régions métropolitaines européennes. Celles-ci constituent les forces majeures d'entraînement tant de l'économie que de la société. Elles ont comme caractéristique essentielle d'être des structures territoriales sans limites, dépassant les contraintes administratives traditionnelles. Sans vouloir présenter un tableau exhaustif des caractéristiques de l'ensemble des régions métropolitaines européennes, les tableaux 1 et 2 présentent les fonctions principales qu'elles assument et schématisent les rapports d'une région métropolitaine avec l'espace international d'une part, avec la région environnante d'autre part. La figure 1 montre cet aspect pour la région métropolitaine de Zurich.

Tableau 1 : Les fonctions principales des métropoles régionales européennes

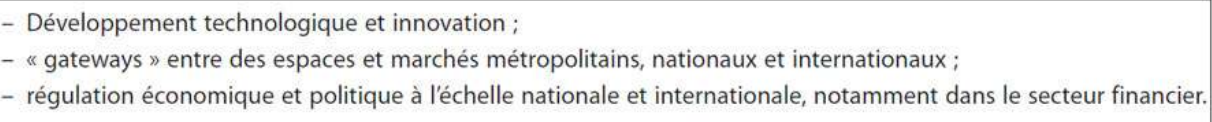


Tableau 2 : Caractéristiques des métropoles régionales européennes

\begin{tabular}{|l|l|}
\hline \multicolumn{1}{|c|}{ Caractéristiques qualitativo-fonctionnelles } & Caractéristiques systémico-structurelles \\
\hline - carrefour européen de transport & $-\begin{array}{l}\text { mise en réseau régionale du noyau urbain } \\
\text { métropolitain dans un système mono/polycentrique } \\
\text { - densité démographique élevée }\end{array}$ \\
- centre économique métropolitaine \\
$\begin{array}{l}\text { internationales } \\
\text { - centre de décision politique et économique }\end{array}$ & $\begin{array}{c}\text { absence d'organisation et d'institutionalisation poli- } \\
\text { tique }\end{array}$ \\
- centre de services et centre financier & - régulation subpolitique par un système d'acteurs \\
- centre de foires & non institutionalisé politiquement \\
- centre médiatique & - apparition de formes de collaboration \\
- centre de recherche-développement, & coopérative entre l'économie et la politique \\
centre scientifique et d'enseignement & par la création de groupements privés \\
- offre culturelle internationale & stimulateurs de politique métropolitaine \\
& - fonction d'interface entre des réseaux \\
& économiques et politiques régionaux, \\
& nationaux et internationaux \\
\hline
\end{tabular}

Source : Blotevogel, 1998

structures polycentriques métropolitaines et des fonctions qui en découlent (Sassen 1991,1994; Andersson et Andersson 2000; Marcuse et van Kempen 2000; Soja 2000 ; Scott 2001). En Allemagne, le concept de régions métropolitaines européennes est intégré dans la politique d'aménagement du territoire, quoiqu'il ne soit accompagné ni de précisions théoriques ni d'indications empiriques (Ritter 1997, Blotevogel 1998, Michel 1998, Motzkus 2000). La Conférence allemande des ministres de l'aménagement du territoire («Ministerkonferenz für Raumordnung - MKRO ») a ajouté au principe classique des lieux centraux le niveau des régions métropolitaines européennes à structure fonctionnelle, en vue d'être à même de réagir plus aisément en matière politique à la dynamique sociétale, économique et culturelle (« Bundesministerium für Raumordnung, Bauwesen und Städtebau, $2000 »)$. Dans d'autres pays européens cependant, cette dernière orientation s'impose visiblement de façon croissante. Le projet «European Metropolitan Regions», conduit dans le sillage de la "Global Conference on the Urban Future (URBAN 21) ", qui s'est tenue en 2000, a donné lieu à l'étude de nombreuses régions métropolitaines d'Europe occidentale et orientale («European Metropolitan Regions Project 1999). L'OCDE a fait un pas de plus et s'est déjà préoccupée de l'indispensable interrogation sur le degré de possibilité d'une gestion politique de telles constructions spatiales (OCDE 2000). Le thème de la gouvernance métropolitaine est capital pour le devenir de la recherche dans le domaine de l'aménagement du territoire. Les développements que nous venons d'esquisser sont-ils aussi valables pour la Suisse? En d'autres termes, des régions métropolitaines européennes sont-elles également identifiables en Suisse?

Notre hypothèse admet qu'une région métropolitaine européenne Zurich est identifiable, dans la mesure où elle remplit les métafonctions évoquées ci-dessus. Il existe en effet à l'intérieur de cette région métropolitaine structurée de façon polycentrique une division du travail horizontale et verticale qui s'acquitte des 
fonctions mentionnées ci-dessus (Behrend et Kruse 2001). Il est toutefois nécessaire de prouver empiriquement et de manière précise dans quelle mesure Bâle constitue l'un des centres majeurs supputé de la région métropolitaine européenne formée autour de Zurich. Insistons néanmoins une nouvelle fois sur le fait que la région métropolitaine européenne de Zurich correspond à un système régional de fonctionnement tout autre que celle de la région bâloise sur le Rhin supérieur.

\section{Essai d'approche empirique}

La fonction de Bâle, à la fois comme "capitale » régionale et comme second noyau majeur de la région métropolitaine européenne de Zurich, devrait en somme être perceptible dans une division du travail différenciée entre Zurich et Bâle, tant horizontalement par une spécialisation réciproque dans des branches déterminées, que verticalement en ce qui concerne le management et la production, respectivement les "back-offices". La globalisation du système financier au cours des dix à quinze dernières années a conduit au fait que dans le monde entier seuls quelques grands pôles se concurrencent encore et que, dans l'optique de cette globalisation, seul un pôle majeur par Etat est perçu comme émergeant au niveau international. Saskia Sassen (1999) suppose que cette situation implique que le pôle financier le plus important de chaque Etat se renforce au détriment de ses concurrents. Ces pôles dominants constituent les "gateways " mondiaux qui assurent les flux réciproques de capitaux entre les Etats : c'est par leur truchement que le capital international pénètre dans un Etat et que le capital national se place à l'étranger ou y fait l'objet d'investissements directs. Selon ce schéma, Zurich devrait avoir augmenté en importance sur le marché financier suisse par rapport à Bâle. La taille métropolitaine, le degré d'internationalisation et l'aéroport sont à cet égard des facteurs-clés. Diverses branches d'activité devraient donner lieu à des analyses analogues, en vue de déterminer des divisions du travail intervenues et, par là-même, le degré de perception de l'internationalisation d'un grand espace économique.

Les auteurs de cet article sont conscients du fait que la preuve empirique d'une appartenance fonctionnelle de Bâle à la mouvance de Zurich est une affaire complexe, qui nécessiterait une ample investigation. Les données disponibles pour l'analyse, telles que par exemple celles de la statistique NOGA $^{1}$, permettent uniquement des identifications et des confrontations de certains indicateurs-clés, destinées à la description de diverses structures bâloises et zurichoises ou à celles à d'autres points nodaux de la région métropolitaine européenne de Zurich. Cette possibilité n'autorise cependant qu'une interprétation, entre ces nœuds majeurs, de possibles divisions du travail. La cohérence fonctionnelle n'est par contre pas prouvée du tout par ces approches très fragmentaires. La plupart des analyses régionales sont d'ailleurs confrontées à ce problème. 


\section{La division du travail dans la région métropolitaine de Zurich}

\section{A. Données d'ensemble}

Présentons d'abord les spécialisations de Bâle et Zurich. Elles sont à même de fournir des indications sur de possibles divisions verticales du travail. Les données utilisées ne se rapportent toutefois qu'à la ville et à l'agglomération des pôles bâlois et zurichois. Elles ont été exploitées en vue de l'obtention d'ensembles de base par rapport à la région métropolitaine de Zurich. La figure 2 montre les quotients de localisation des agglomérations de Zurich et de Bâle, ainsi que des noyaux urbains, d'un certain nombre de branches d'activité significatives.

Figure 2 : Quotients de localisation selon le nombre d'actifs des villes et des agglomérations de Zurich et de Bâle en 1998, par rapport à la région métropolitaine de Zurich

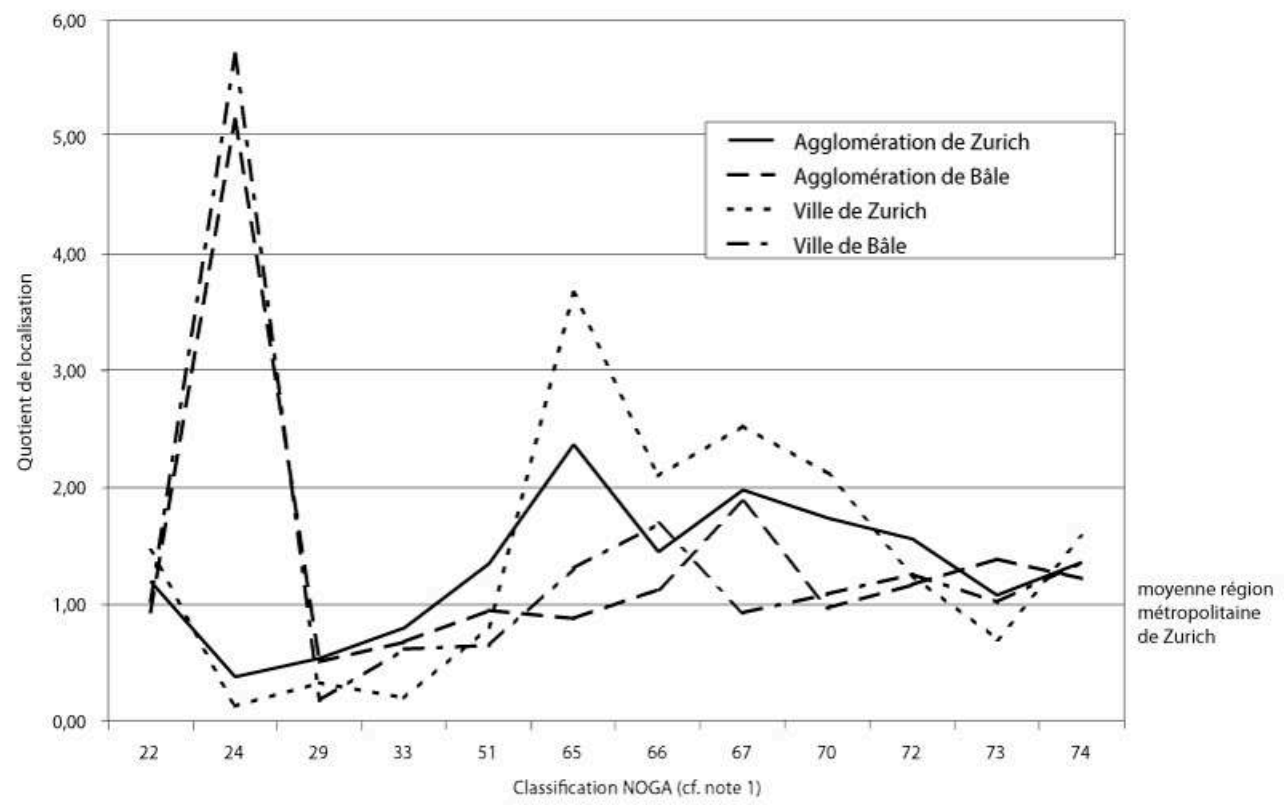

La spécialisation extrême de l'industrie chimique bâloise, convertie entre-temps radicalement en industrie pharmaceutique, est particulièrement impressionnante (NOGA 24). Mais les autres branches de la production sont situées au-dessous du degré de concentration moyen observé dans la région métropolitaine (moyenne $=1$ ), à l'exception de l'édition et de l'impression qui sont fortement dépendantes des médias et des services. Les branches tertiaires par contre sont positionnées au-dessus de cette moyenne, avec toutefois des degrés de concentration variés selon les branches. Dans le domaine du crédit (NOGA 65) la prédominance de Zurich saute aux yeux : ici le nombre d'emplois est trois fois et demi plus élevé que la moyenne de la région métropolitaine. Le niveau est également très élevé dans l'agglomération de Zurich, noyau urbain inclus. Cet écart imposant s'explique par le fait que la ville de Zurich concentre à elle seule de nombreux services centraux. La concentration bâloise cependant ne dépasse guère la moyenne de la région métropolitaine. Il est particulièrement intéressant d'observer la répartition des activités liées au crédit et aux assurances (NOGA 67). Ici l'agglomération de Bâle atteint une concentration plus élevée que la ville de Bâle. Ce phénomène peut 
s'expliquer par une certaine spécialisation en matière d'activités secondaires dans la branche du crédit. La concentration relativement faible de services à Bâle est en fait conditionnée par la nature de l'évaluation: le nombre très élevé d'actifs dans l'industrie chimique minimise les parts constituées par les services en comparaison avec la région métropolitaine. Cette précision devrait écarter toute supposition selon laquelle Bâle ne disposerait pas d'une offre complète de services correspondant à sa fonction de métropole régionale.

Des spécialisations horizontales sont également à relever dans d'autres agglomérations de la région métropolitaine de Zurich. Quatre d'entre elles ont servi de base à l'élaboration de la figure 3.

Figure 3 : Quotients de localisation, selon le nombre d'actifs en 1998, des villes de Zug, Winterthur, Saint-Gall et Olten, par rapport à la région métropolitaine de Zurich

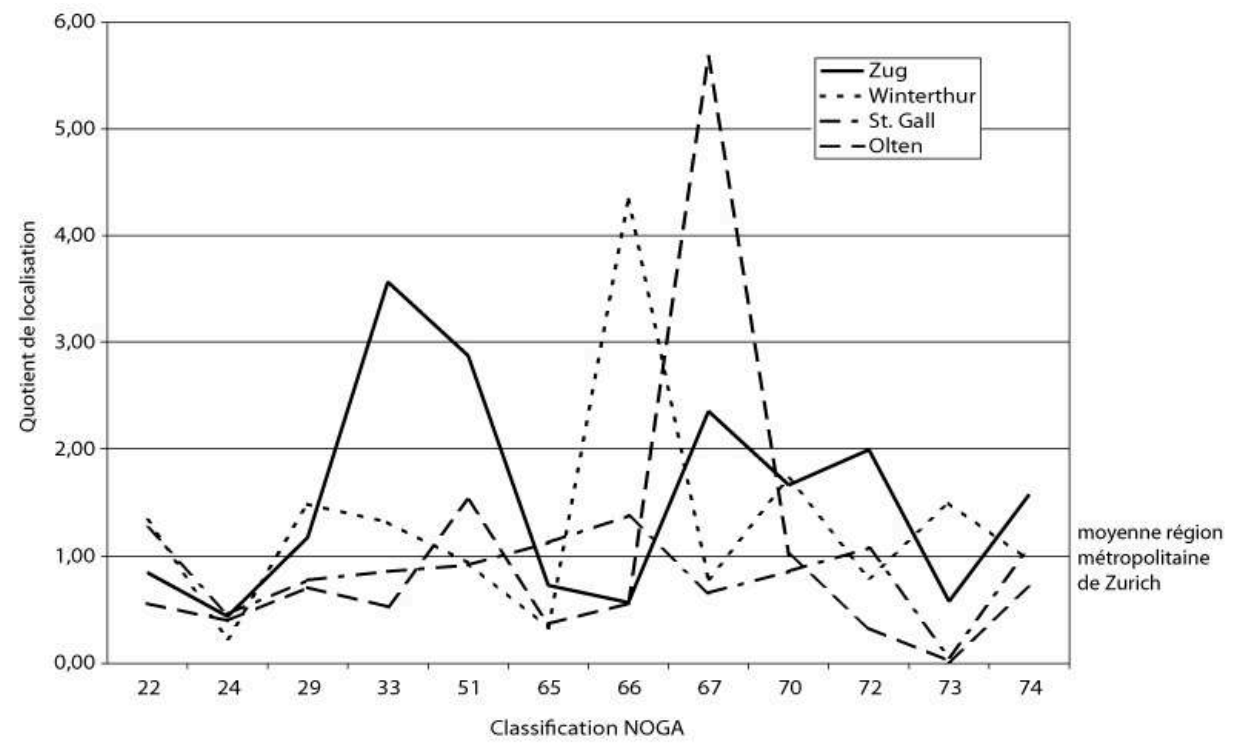

Classes NOGA 22 à 74 : cf. note 1.

Sources : Bundesamt für Statistik, Arbeitsstättenzählung, calculs des auteurs.

La figure 3 révèle qu'avec des valeurs deux fois supérieures à la moyenne de la région métropolitaine, l'agglomération de Zug est fortement spécialisée dans les quatre branches suivantes : la production d'instruments médicaux et de précision (NOGA 33), le commerce de gros et le courtage commercial (holdings commerciaux et traditionnel commerce important de matières premières), les activités liées au crédit et aux assurances (à Zug surtout la gestion patrimoniale) et les services informatiques. L'agglomération de Winterthur est caractérisée par une forte spécialisation dans les assurances, par suite de la présence des Assurances Winthertur. La spécialisation la plus importante, avec un quotient de localisation de 5,68, est celle d'Olten, dans la branche « activités liées au crédit et aux assurances ». A l'opposé des spécialisations de nature plus historique de Zug et de Winterthur, celle d'olten est nouvelle ; elle est soustendue par la firme SEGA-Intersettle («Clearing \& Settlement»), par ailleurs localisée à Zurich. L'agglomération de Saint-Gall témoigne d'une autre image: elle n'est pas spécialisée dans une branche déterminée, phénomène qui pourrait s'expliquer par la plus grande distance par rapport à Zurich, contrairement aux trois autres agglomérations : les spécialités très variées et équilibrées font la preuve d'une forte 
autonomie de Saint-Gall en tant que centre régional, tant en ce qui concerne sa taille que pour ce qui est de son rôle. Naguère, Saint-Gall a été le centre d'industrie textile hautement spécialisé le plus marquant de la Suisse. A présent, celle-ci est en pleine mutation ; seuls quelques segments hautement productifs subsistent.

Compte tenu de ce qui précède, il est possible de faire les constatations suivantes, au moins pour les régions métropolitaines polycentriques : des noyaux de l'espace autour du centre métropolitain présentent des spécialisations dans certaines branches; ces spécialisations sont fondées sur une division horizontale du travail requérant des conditions de localisation différenciées. Les sociétés de holding sont par exemple intéressées entre autres par des impôts locaux avantageux et la faible distance qui les sépare de Zurich ou de l'aéroport ; pour les «back-offices »- les fonctions banales des services financiers, bancaires et ceux des assurances - l'accessibilité grâce à des axes de communication appropriés, ainsi que le prix du foncier, sont déterminants.

\section{B. Le développement de la division du travail}

24 La figure 4 indique l'évolution des quotients de localisation de branches d'activités de services typiques dans les villes de Zurich et de Bâle entre 1985 et 1998. Elle illustre une réduction de la concentration de ces activités dans les villes, vraisemblablement et surtout par suite de leur accroissement dans les communes agglomérées autour des villes ou de leur transfert dans ces communes. Dans le crédit et les assurances (NOGA 65 et 66), l'augmentation de la concentration se présente différemment à Bâle et à Zurich. Dans la ville de Bâle elle a augmenté autrement qu'à Zurich, tant dans les services informatiques (NOGA 72) que dans la recherche-développement (NOGA 73). De très fortes réductions ont eu lieu à Zurich dans les activités liées au crédit et aux assurances; il en est ainsi à Bâle et à Zurich en ce qui concerne la location d'objets mobiles (NOGA 71), le tout ayant été sans doute suscité par les coûts élevés des surfaces de plancher en centre-ville. A Zurich, l'augmentation de la concentration dans le crédit et les assurances témoigne en premier lieu de l'ampleur prise par les sièges sociaux principaux des firmes, en second lieu de l'importance des lieux centraux pour des clients privés fortunés et entrepreneuriaux. Pour la desserte de ce type de clientèles, le prestige et l'environnement sont plus déterminants que les coûts occasionnés par la localisation. Cette constatation est également valable pour toute une série de services aux entreprises (NOGA 94): dans ce domaine, la concentration qui s'est opérée entre 1985 et 1998, au moment d'une forte croissance de la branche, ne s'est guère modifiée entre-temps ; pour la ville de Bâle, elle se situe toutefois au-dessous de la moyenne de la région métropolitaine. Une évolution semblable s'est réalisée dans les branches relatives aux groupements d'intérêts et aux associations (NOGA 91), ainsi que dans celle intitulée " distractions, culture, sports " (NOGA 92); dans les deux cas, la préférence accordée à une forte centralité apparaît clairement. 
Figure 4 : Modification des quotients de localisation de branches d'activités typiques dans les villes de Zurich et de Bâle entre 1985 et 1998

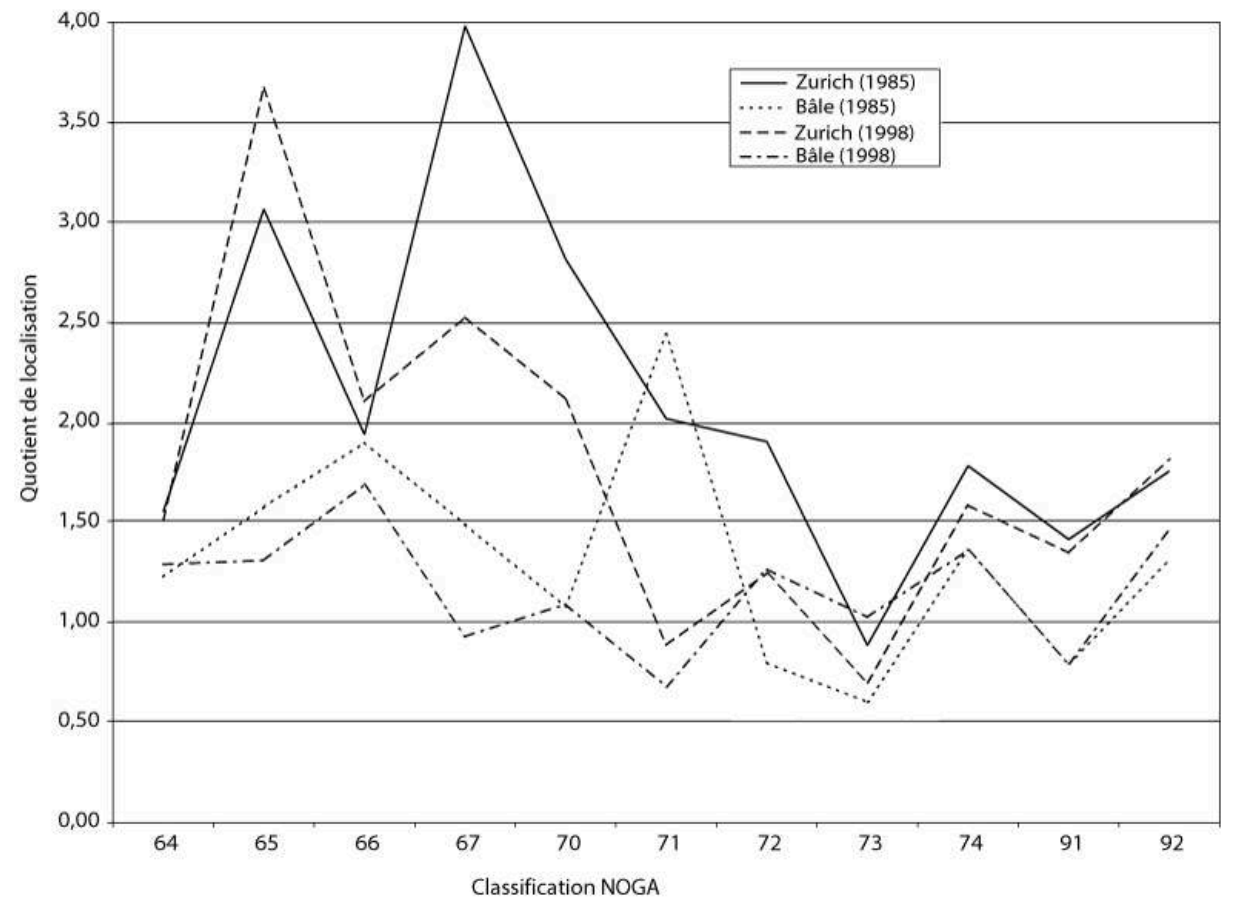

Note : identification des classes NOGA 64 à 92 : cf. note 1.

Sources : Bundesamt für Statistik, Arbeitsstättenzählung, calculs des auteurs.

La diminution du quotient de localisation ne signifie pas qu'il y ait eu également perte d'emplois, car la mutation structurelle s'est opérée dans la région métropolitaine étudiée prise dans son ensemble. La figure 5 montre comment s'est déroulée l'évolution de l'emploi dans les villes de Zurich et de Bâle. Nous apercevons nettement la mutation de branches productives en services; l'essentiel de cette mutation a porté sur le commerce de gros et de détail (NOGA 51,52); elle a été fondée sur le transfert en agglomération, ainsi que sur la rationalisation. Dans cette mutation, Zurich a enregistré davantage de pertes relatives que Bâle. Comme Zurich a un nombre d'emplois plus de deux fois supérieur à celui de Bâle, il convient, à des fins de comparaison, de doubler l'importance de Bâle dans l'interprétation de la figure 5. Avec cet éclairage, le recul de l'emploi dans le secteur productif a été plus important à Zurich qu'à Bâle. Dans le secteur des services, la mutation structurelle a aussi des répercussions plus amples à Zurich qu'à Bâle. Tandis que Bâle a connu une croissance plus forte que Zurich dans les technologies de l'information (NOGA 72) et les services aux entreprises (NOGA 74), cette évolution est compensée supérieurement à Zurich par la croissance d'autres services. Relevons à nouveau le crédit (NOGA 65) qui a enregistré à Zurich une augmentation de plus de 6000 emplois, alors que Bâle y a perdu plus de 1300 emplois. Cette décroissance est sans doute essentiellement inhérente à la fusion de l'Union de Banques Suisses (UBS) avec la "Schweizer Bank» (Banque Suisse), opération par laquelle Bâle a perdu beaucoup en importance. Quoique l'USB affiche que Bâle reste le lieu de son second siège, une part déterminante des activités de niveau supérieur a été transférée à Zurich en vue d'effets synergétiques potentiels. 
Figure 5 : Evolution des emplois dans des branches d'activités significatives à Zurich et à Bâle entre 1985 et 1998

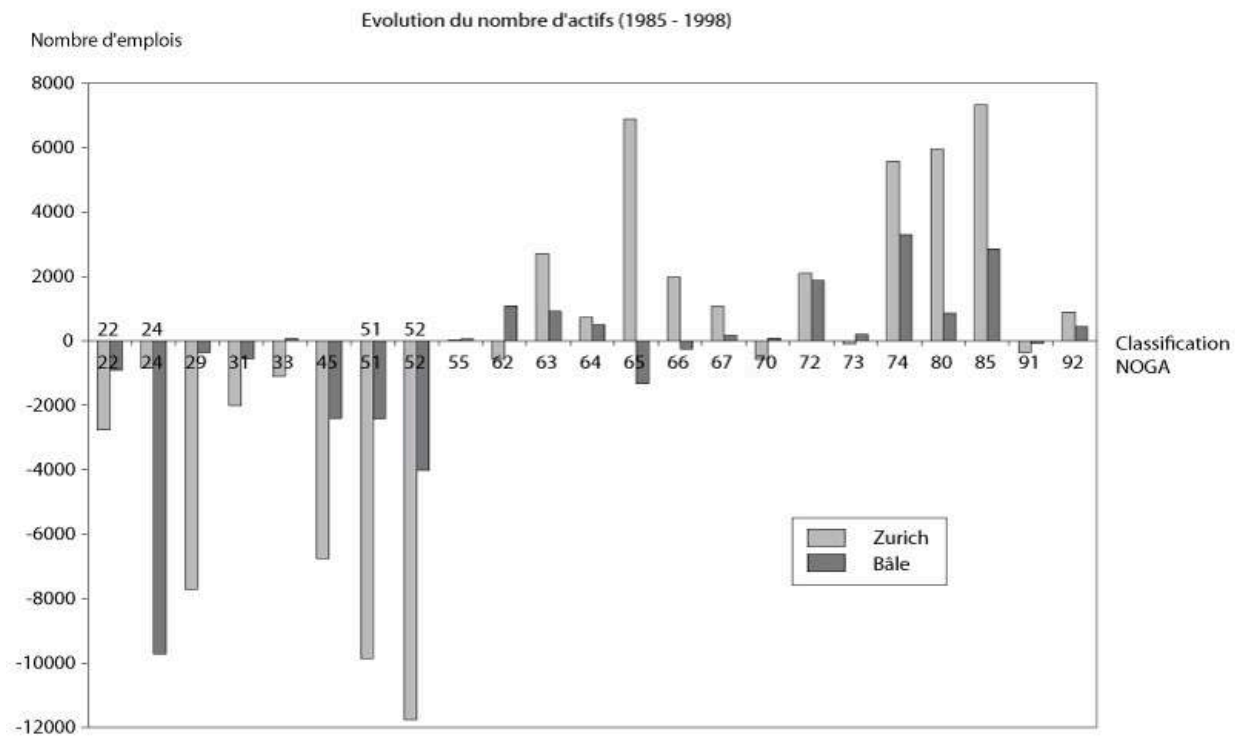

Note : identification des classes NOGA de 22 à 92 : cf. note 1.

Sources : Bundesamt für Statistik, Arbeitsstättenzählung, calculs des auteurs.

L'augmentation de l'importance de Zurich en tant que place financière prépondérante à l'échelle suisse conforte l'hypothèse de Saskia Sassen (1999), selon laquelle la globalisation ne permet plus à un Etat de posséder davantage qu'un pôle à vocation mondiale dans une activité déterminée, autour duquel celle-ci se concentre désormais nécessairement. Cette affirmation est certes atténuée par la forte concurrence exercée par Genève, avant tout dans le domaine de la clientèle privée. Dès lors que l'on considère cependant le chiffre d'affaires des banques, selon leur localisation géographique, Zurich est le grand gagnant entre 1993 et 1999, avec le gain d'une part de marché de l'ordre de $23,7 \%$. Saint-Gall a pu grignoter 1,6\% de part de marché, avec la « Raiffeisenbank » (de type Crédit Mutuel); toutes les autres villes ont soit maintenu un tant soit peu leur niveau, soit perdu en importance : c'est notamment le cas de Bâle, avec une perte de $15 \%$ (par le transfert du chiffre d'affaires de la "Schweizer Bank ", par fusion, à Zurich), et de Berne, avec une perte de 3,7 \%; Lausanne et les autres villes ont perdu au total 5,6\%. A présent le marché financier suisse se concentre à Zurich, qui détient 76,7 \% des parts de marché helvétiques contre 53,0 \% en 1993 (tableau 3). 
Tableau 3 : Chiffre d'affaires des banques sur les principales places financières suisses et évolution des parts de marché

\begin{tabular}{|c|c|c|c|c|c|c|}
\hline \multirow{3}{*}{$\begin{array}{c}\text { Place } \\
\text { financière }\end{array}$} & \multicolumn{4}{|c|}{ Chiffre d'affaires } & \multirow{2}{*}{\multicolumn{2}{|c|}{$\begin{array}{c}\text { Evolution du chiffre d'affaires } \\
\text { 1993-1999 }\end{array}$}} \\
\hline & \multicolumn{2}{|c|}{1993} & \multicolumn{2}{|c|}{1999} & & \\
\hline & en millions FS & $\begin{array}{l}\text { en } \% \text { total } \\
\text { de la Suisse }\end{array}$ & en millions FS & $\begin{array}{l}\text { en } \% \text { total } \\
\text { de la Suisse }\end{array}$ & en $\%$ & $\begin{array}{l}\text { en écarts de } \\
\text { points (\%) }\end{array}$ \\
\hline Zurich & 753658 & 53,0 & 1917691 & 76,7 & 154,5 & 23,7 \\
\hline Genève & 76643 & 5,4 & 125882 & 5,0 & 64,2 & $-0,4$ \\
\hline Saint-Gall & 28365 & 2,0 & 89650 & 3,6 & 216,1 & 1,6 \\
\hline Lausanne & 34247 & 2,4 & 36396 & 1,5 & 6,3 & $-0,9$ \\
\hline Berne & 72115 & 5,1 & 36142 & 1,4 & $-49,9$ & $-3,7$ \\
\hline Lugano & 15861 & 1,1 & 35262 & 1,4 & 122,3 & 0,3 \\
\hline Bâle & 231565 & 16,3 & 33746 & 1,3 & $-85,4$ & $-15,0$ \\
\hline Diverses & 208439 & 14,7 & 226855 & 9,1 & 8,8 & $-5,6$ \\
\hline Total & 1420892 & 100,0 & 2501624 & 100,0 & 76,1 & \\
\hline
\end{tabular}

Sources : Behrendt, Kruse, 2001 ; Données : «Handelszeitung »; calculs personnels des auteurs.

\section{Perspectives}

Quel est le degré d'expressivité des données présentées ci-dessus ? Elles ont au moins le mérite de faire ressortir clairement un aspect important: la mutation structurelle observée dans diverses branches d'activités au cours des dernières années est un phénomène international, auquel la localisation prise individuellement est exposée et par rapport auquel elle doit se positionner. C'est une question d'échelle. Dans le contexte de la dimension de la métropole régionale européenne de Zurich, il n'est pas indiqué, surtout pas politiquement, de mettre par exemple l'accent sur une compétition malsaine entre Bâle et Zurich. Bâle est certainement le noyau d'une région trinationale $\mathrm{du}$ Rhin supérieur; dans le contexte du système des régions métropolitaines européennes, des portions de cette région forment toutefois un noyau important de la région métropolitaine européenne de Zurich. Il n'est donc pas opportun d'adopter le point de vue selon lequel ces lieux sont susceptibles de s'approprier réciproquement des fonctions dans une vaine concurrence. Il y a eu certes - et les données statistiques l'ont montré - différents décalages. Mais considérées à l'échelle internationale, des spécialisations identifiables de carrefours témoignent de la nécessité d'une collaboration à l'intérieur des régions métropolitaines. Il est ainsi d'un très grand intérêt de s'interroger sur la façon dont ces spécialisations peuvent être mises en réseau.

28 La tentative de concentrer ces spécialisations dans le cadre de la "Greater Zurich Area » (GZA) et la manière neutre de considérer toute implantation d'entreprise dans l'un des pôles comme un gain pour l'ensemble de l'espace économique de Zurich, sont des signes de reconnaissance de la perception de tels avantages à l'échelle internationale. Des initiatives analogues existent en fin de compte depuis plusieurs années dans de nombreuses régions métropolitaines européennes. Elles doivent entrer nécessairement en conflit avec les institutions politiques. Elles sont liées à de nombreux problèmes et questions qui requièrent inévitablement une affirmation politique. C'est pour cette raison que de nombreuses régions, ainsi que des organisations internationales, s'interrogent déjà sur la gouvernance métropolitaine (OCDE, 2000). La difficulté réside dans le fait que cette thématique se heurte aux piliers 
même de l'organisation politique et territoriale des Etats nationaux actuels. L'existence du système suisse de réseaux urbains, confirmée par le parlement, et partie prenante des bases fondamentales de l'aménagement du territoire de la Suisse (« Bundesamt für Raumplanung " 1996, Office fédéral de l'aménagement du territoire), montre que la division du travail entre pôles a été aussi perçue politiquement. La réflexion sur les répercussions de celle-ci demeure cependant à l'état embryonnaire.

Si la question de savoir dans quelle mesure Bâle relève de Zurich n'a certainement pas trouvé de réponse satisfaisante dans le présent article, quelques indices témoignent néanmoins de l'existence d'une division du travail horizontale et verticale.

Les expériences frontalières, mentales et physiques, doivent être encore renforcées; des échelles nouvelles doivent être définies. Pour le discours scientifique en Suisse - et pas seulement pour lui -, il importe de se familiariser de façon plus intense avec les structures des régions métropolitaines, de même qu'avec les approches méthodologiques destinées à permettre d'analyser les systèmes fonctionnels dans leurs contextes spatiaux. Les sciences de l'espace ne peuvent pas se dérober à leur devoir d'élaboration de concepts permettant de prendre en compte de nouveaux systèmes territoriaux.

\section{BIBLIOGRAPHIE}

ANDERSSON A., ANDERSSON D.E. (sous la direction de) (2000). - Gateways to the Global Economy, Cheltenham, Northampton : Edward Elgar.

BEHRENDT H., KRUSE C. (2001). — « Die Europäische Metropolregion Zürich - die Entstehung des subpolitischen Raumes ». Geographica Helvetica, 3/56:1-12.

BonNEVILLE M. (1994) - «Internationalization of Non-capital Cities in Europe : Aspects, Processes and Prospects ». European Planning Studies, 2.3 : 267-285.

Blotevogel H.H. (1998). - Europäische Metropolregion Rhein-Ruhr. Theoretische, empirische und politische Perspektiven eines neuen raumordnungspolitischen Konzepts. Institut für Landes- und Stadtentwicklungsforschung, 135. Dortmund.

BUNDESAMT FÜR RAUMPLANUNG (1996) - Die Grundzüge der Raumordnung Schweiz, Berne. BUNDESMINISTERIUM FÜR RAUMORDNUNG, BAUWESEN UND STÄDTEBAU (2000). - « Raumordnungsbericht $2000 »$. Berichte, 7. Bonn.

Dielemann F.M., Faludi A. (1998). - « Randstad, Rhine-Ruhr and Flemish Diamond as One Polynucleated Macro-region?». Tijdschrift voor Economische en Sociale Geografie, $89.3: 320-327$.

EUROPEAN METROPOLITAN REGIONS PROJECT (1999). — « Strategies for Sustainable Development of European Metropolitan Regions. Evaluation Report. Submitted to the European Regional Conference „European Metropolitan Regions », Essen,15. -17. September 1999.

Handelszeitung (1994). - Top 1994. Die grössten Unternehmen der Schweiz, Zurich. 
HANDELSZEITUNG (2000). - Top 2000. Die grössten Unternehmen der Schweiz, Zurich.

MARcuse P., van Kempen R. (sous la direction de) (2000). - Globalizing Cities. A New Spatial Order?, Oxford : Malden.

Michel D. (1998). - « Das Netz der europäischen Metropolregionen in Deutschland ». Raumforschung und Raumordnung, 56.5/6:362-367.

Motzkus A. (2000). - « Zur Bedeutung der höherwertigen unternehmensorientierten Dienstleistungen für die Entwicklung der Metropolregionen Westdeutschlands ». Raumforschung und Raumordnung, 58.4 : 265-275.

OCDE (2000). - Cities for Citizens. Improving Metropolitan Governance. Working Party on Territorial Policy in Urban Areas. Report DT/TDPC/URB (2000) 4. Paris.

RECLUS (1989). - Les villes européennes - Rapport pour la Datar, La Documentation française, Paris.

RITTER E.H. (1997). - « Europäische Metropolregion Rhein-Ruhr oder : Kann aus einer StädteAgglomeration eine Metropolregion werden?».Forschungs- und Sitzungsberichte der Akademie für Raumforschung und Landesplanung, $202:$ 156-170.

SASSEN S. (1991). - The Global City : New York, London, Tokyo. Princeton.

SASSEN S. (1994). - Cities in a World Economy. London.

SASSEN S. (1999). - « Global Financial Centers ». Foreign Affairs, 78 : 75-87.

ScotT A. (sous la direction de) (2001). - Global-City-Regions : Trends, Theory, Policy. Oxford : Oxford University Press.

SojA E.W. (2000). - Postmetropolis. Critical Studies of Cities and Regions. Oxford, Malden.

zÜRCHER NATIONALBANK (2000). - Metropole Zürich. Der Wirtschaftsstandort im

Vergleich. Zürich.

\section{NOTES}

1. La Nomenclature générale des activités économiques (NOGA) a remplacé en 1995 celle de 1985 relative à la Systématique générale des branches économiques. Elle correspond à son pendant de l'Union européenne, NACE (Nomenclature générale des activités économiques dans les Communautés Européennes) Rev.1-Eurostat. Celle-ci s'appuie sur l'ordonnance 696/93 relative aux Unités statistiques destinées à «l'observation et à l'analyse de l'économie dans l'Union européenne » (Bulletin officiel des Communautés européennes L76 du 30 mars 1993).

Sources: Office Fédéral de la Statistique, recensement des lieux de travail, calculs des auteurs Classes NOGA utilisées pour les figures 2 à 5 :

22 Edition et impression

24 Industrie chimique

29 Construction mécanique

31 Fabrication d'appareils pour la production d'électricité

32 Fabrication d'appareils médicaux, instruments de précision, appareils d'optique, montres

45 Construction

51 Commerce de gros et médiation commerciale

52 Commerce de détail et réparations 
55 Hôtellerie et restauration

62 Aviation

63 Auxiliaires de transport et activités annexes

64 Télécommunications

65 Crédit

66 Assurances

67 Activités liées au crédit et aux assurances

70 Immobilier

72 Informatique

73 Recherche-développement

74 Services aux entreprises

80 Enseignement

85 Santé et action sociale

91 Représentation d'intérêts et associations

92 Distractions, culture et sport

\section{RÉSUMÉS}

La présente contribution s'appuie sur l'existence effective d'une région métropolitaine européenne de Zurich dont les fonctions multiples s'affranchissent des limites administratives et qui intègre l'agglomération de Bâle. Préciser les rôles respectifs de Zurich et de Bâle suppose au préalable l'identification de la division horizontale et verticale du travail, résultant des localisations d'entreprises et des acteurs en présence. Comme toutefois les données statistiques demeurent insuffisantes et que la démarche empirique adoptée est difficile et complexe, un discours politique ne saurait être qu'imprécis et stéréotypé. Il serait donc urgent d'avoir recours à de nouvelles formes de gouvernance métropolitaine prenant en compte la dynamique urbaine inhérente aux nouvelles spécialisations et orientations économiques.

This contribution supposes the existence of an European Metropolitan Region of Zurich, which is - detached by administrative boundaries - functionally structured and operating on the level of the agglomeration. The study about the respective role of Zurich and Basle presupposes however the identification of an horizontal and vertical division of labour of locations and actors. Since this is empirically difficult and complex, clear evidences are missing. Therefore an imprecise and stereotyped political discourse can be constituted. The new economic dynamic make urgently necessary to search for new forms of policies in the context of metropolitan governance.

Dieser Beitrag geht von der Existenz einer Europäischen Metropolregion Zürich aus, die losgelöst von administrativen Grenzen funktional strukturiert ist und auf der Ebene der Agglomeration operiert. Die jeweilige Rolle von Zürich und Basel festzustellen setzt allerdings die Identifizierung einer horizontalen und vertikalen Arbeitsteilung von Standorten und Akteuren voraus. Da dies empirisch schwierig und komplex ist und somit eindeutige Belege fehlen, lässt sich auch nur ein unpräziser und stereotyper politischer Diskurs ausmachen. Dabei wäre es dringend notwendig, sich mit neuen Politikformen der metropolitan governance auseinanderzusetzen, welche die neue wirtschaftliche Dynamik einbezieht. 
INDEX

Mots-clés : analyse régionale, gouvernance métropolitaine, région métropolitaine européenne de Zurich, régions polycentriques, systèmes fonctionnels

Schlüsselwörter : Europäische Metropolregion Zürich, funktionale Systeme, metropolitan governance, polyzentrische Regionen, Regionalanalyse

Keywords : European Metropolitan Region Zurich, functional systems, metropolitan governance, polycentric regions, regional analysis

\section{AUTEURS}

\section{HEIKO BEHRENDT}

Institut für Orts-, Regional- und Landesplanung (ORL) - Eidgenössische Technische Hochschule Zurich (ETH) - CH-8093 ZÜRICH - Behrendt@orl.arch.ethz.ch

\section{CHRISTIAN KRUSE}

Institut für Orts-, Regional- und Landesplanung (ORL) - Eidgenössische Technische Hochschule Zurich (ETH) - CH-8093 ZÜRICH - Kruse@orl.arch.ethz.ch 